\title{
Optimal Model-Free Output Synchronization of Heterogeneous Systems Using Off-Policy Reinforcement Learning
}

\author{
H. Modares ${ }^{\mathrm{a}}$, S. P. Nageshrao ${ }^{\mathrm{b} *}$, G. A. D. Lopes ${ }^{\mathrm{b}}$, R. Babuška ${ }^{\mathrm{b}}$, and F. L. Lewis ${ }^{\mathrm{a}, \mathrm{c}}$ \\ * Corresponding author. Phone +31152788573 Fax+31152786679 email s.p.nageshrao@tudelft.nl \\ ${ }^{a}$ University of Texas at Arlington Research Institute, 7300 Jack Newell Blvd. S., Ft. Worth, TX 76118, USA \\ ${ }^{b}$ Delft Center for Systems and Control, Delft University of Technology, Mekelweg 2, 2628 CD, Delft, The Netherlands \\ ${ }^{c}$ Qian Ren Consulting Professor, State Key Laboratory of Synthetical Automation for Process Industries, Northeastern University,
} Shenyang, China

\begin{abstract}
This paper considers optimal output synchronization of heterogeneous linear multi-agent systems. Standard approaches to output synchronization of heterogeneous systems require either the solution of the output regulator equations or the incorporation of a p-copy of the leader's dynamics in the controller of each agent. By contrast, in this paper neither one is needed. Moreover, here both the leader's and the follower's dynamics are assumed to be unknown. First, a distributed adaptive observer is designed to estimate the leader's state for each agent. The output synchronization problem is then formulated as an optimal control problem and a novel model-free off-policy reinforcement learning algorithm is developed to solve the optimal output synchronization problem online in real time. It is shown that this optimal distributed approach implicitly solves the output regulation equations without actually doing so. Simulation results are provided to verify the effectiveness of the proposed approach
\end{abstract}

Key words: Output synchronization, Heterogeneous systems, Reinforcement Learning, Leader-follower systems

\section{Introduction}

Cooperative control of multi-agent systems has undergone a paradigm shift from centralized to distributed, due to its reliability, flexibility, scalability and computational efficiency. In distributed control, each agent designs a controller based on limited information about itself and its neighbors to assure all agents reach agreement on certain quantities of interests (leaderless consensus) or all agents follow trajectories of a leader node (leaderfollower control). A rich body of literature has been developed on distributed control of multi-agent systems. See for example (Jadbabaie, Lin \& Morse, 2003, Olfati-Saber \& Murray, 2004, Lewis et al., 2014, Ren, Beard \& Atkins, 2007, Ren \& Beard, 2008) to name a few.

Most of the existing work on distributed control focuses on state synchronization of homogeneous multi-agent systems with identical dynamics. Distributed output synchronization of heterogeneous systems, where the dynamics and dimension of agents can be different, has also attracted attention (Lunze, 2012; Wieland, Sepulchre \& Allgöwer, 2011; Huang \& Chen, 2004; De Persis \& Jayawardhana, 2014; Yang, et al., 2014; Peymani, et al., 2014; Huang, et al., 2013; Xiang, Wei, \& Li, 2009). Existing mentioned methods, however, require complete knowledge of the agents and the leader's dynamics which is not available in many real-world applications.

Adaptive distributed controllers have been developed to cope with system uncertainties in the dynamics of the agents (Das \& Lewis, 2010; Ding 2015). Adaptive methods can only guarantee a bounded synchronization error and not asymptotic synchronization. This is because existing adaptive methods do not solve the output regulation equations, which is a necessary and sufficient condition to assure perfect synchronization. Moreover, they do not converge to an optimal distributed solution. Robust controllers, on the other hand, require the nominal model of the agents and full knowledge of the leader dynamics. Suboptimal and optimal distributed controllers are also designed in (Zhang, Lewis, \& Das, 2011; Vamvoudakis, Lewis, \& Hudas, 2012; Cao, \& Ren, 2010; Zhang, Feng, Liang, \& Luo, 2015) for linear homogenous systems. These methods, however, are limited to state synchronization and they require complete knowledge of the agents and the leader. To our knowledge distributed adaptive optimal output synchronization is not considered in the literature.

Over the last decades there has been increasing interest in developing the multi-agent learning systems so as to create agents that can learn from experience how to interact with other agents in a best possible way (Lakshmanan \& Farias, 2006; Chang, 2009; Busoniu, Babuska \& De Schutter, 2008). Reinforcement learning (RL) techniques have been used as promising methods to design adaptive optimal controllers for both single-agent and multi-agent systems.

In this paper, a reinforcement learning (RL) algorithm is developed to solve the output synchronization of heterogeneous systems. It is shown that the explicit solution to the output regulator equation is not necessary, hence the agents do not need to know the leader's dynamics. The key components of the given method are:

1) Optimality is explicitly imposed in solving the output synchronization problem. This allows the use of RL to solve the problem in real time.

2) A novel off-policy RL algorithm is developed to solve the output synchronization problem online in real time without requiring any knowledge of the agent's or the leader's dynamic .

3) It is shown that this distributed RL approach implicitly solves the output regulation equations without actually solving them. 
4) A model-free distributed adaptive observer is designed to estimate the leader's state.

The distributed observer gives the feedforward state, which is used along with the local feedback state of each agent to design a local control protocol. A local discounted performance function is defined for each agent, its minimization gives both feedback and feedforward controllers. Online solution to the minimization problem is then found by using an off-policy RL algorithm. This algorithm does not require any knowledge of the dynamics of the agents and uses only the measured data of the system and the reference trajectories to find the optimal distributed solution to the output synchronization problem.

\section{Theoretical background}

In this section, the essential theoretical background on graph theory is provided. The problem of output synchronization for heterogeneous multi-agent systems is also defined.

\subsection{Graph Theory}

A weighted directed graph (digraph) is defined as $\mathcal{G}=(\mathcal{V}, \mathcal{E}$, $A)$, where $\mathcal{V}=\left(v_{1}, v_{2}, \ldots, v_{N}\right)$ is a set of $N$ nodes, $\varepsilon \subset \mathcal{V}$ $\times \mathcal{V}$ is a set of edges, and $A=\left[a_{i j}\right] \in \mathbb{R}^{N \times N}$ is the associated adjacency matrix with $a_{i j}>0$ only if $\left(v_{j}, v_{i}\right) \in \varepsilon$, and $a_{i j}=0$ otherwise. The neighbor set of node $i$ is depicted by $N_{i}=\left\{j \mid\left(v_{j}, v_{i}\right) \in \varepsilon\right\}$. The in-degree of a node $i$ is defined as $d_{i}=\sum_{j=1}^{N} a_{i j}$ and in-degree matrix as $D=\operatorname{diag}\left[d_{i}\right] \in \mathbb{R}^{N \times N}$

. The graph Laplacian matrix is defined as $L=D-A$. A graph is said to be undirected if the graph Laplacian is symmetric, i.e, $L=L^{T}$. For a given digraph $\mathcal{G}$, a sequence of successive edges in the form $\left(\left(v_{i}, v_{k}\right),\left(v_{k}, v_{l}\right), \cdots\left(v_{m}, v_{j}\right)\right)$ gives a directed path from node $i$ to node $j$. A diagraph is said to have a spanning tree if there exist a root node $i_{r}$, such that there is a directed path from $i_{r}$ to every other node in the graph. A weighted directed graph is called detail balanced if there exist scalars $p_{i}>0, p_{j}>0$ such that $p_{i} a_{i j}=p_{j} a_{j i}$ for all $i, j \in N$ (Lewis et al., 2014).

Assumption 1: The digraph $\mathcal{G}$ has a spanning tree and the leader is pinned to at least one root node.

The leader can be pinned to multiple nodes in the graph. This results in a diagonal pinning matrix $G=\operatorname{diag}\left[g_{i}\right] \in \mathbb{R}^{N \times N}$ with $g_{i}>0$ if the node has access to the leader, and zero otherwise. Under the above assumption, the eigenvalues of $L+G$ have positive real parts (Hong, Chen, \& Bushnell, 2008; Hong, Hu, \& Gao, 2008; Li et al., 2008; Grip et al., 2012).

\subsection{Output Synchronization of Heterogeneous Multi- agent Systems}

Let the leader dynamics be given by

$$
\dot{\zeta}_{0}=S \zeta_{0}
$$

where $\zeta_{0} \in \mathbb{R}^{p}$ is the reference trajectory to be followed by followers, and $S \in \mathbb{R}^{p \times p}$ is the leader's dynamic matrix.
Using the output matrix $R \in \mathbb{R}^{q \times p}$, the output of the leader $y_{0} \in \mathbb{R}^{q}$ is

$y_{0}=R \zeta_{0}$

Assumption 2: All eigenvalues of the leader dynamic $S$ are on the imaginary axis and they are non-repeated.

The dynamics of $\mathrm{N}$ linear heterogeneous agents is given by

$$
\begin{aligned}
& \dot{x}_{i}=A_{i} x_{i}+B_{i} u_{i} \\
& y_{i}=C_{i} x_{i}
\end{aligned}
$$

where $x_{i} \in \mathbb{R}^{n_{i}}$ is the system state, $u_{i} \in \mathbb{R}^{m_{i}}$ is the input and $y_{i} \in \mathbb{R}^{q}$ is the output for agent $i$.

Assumption 3: $\left(A_{i}, B_{i}\right)$ is stabilizable and $\left(A_{i}, C_{i}\right)$ is observable.

Problem 1 (Output Synchronization): Design local control protocols $u_{i}$ such that the outputs of all agents synchronize to the output of the leader node. That is, $y_{i}(t)-y_{0}(t) \rightarrow 0, \forall i$.

To solve Problem 1, standard methods in the literature require solving the output regulation equations given by

$$
\begin{aligned}
& A_{i} \Pi_{i}+B \Gamma_{i}=\Pi_{i} S \\
& C_{i} \Pi_{i}=R
\end{aligned}
$$

where $\Pi_{i} \in \mathbb{R}^{n_{i} \times p}$ and $\Gamma_{i} \in \mathbb{R}^{m_{i} \times p}$ for $i=1, \cdots, N$ are solutions to (4). Based on these solutions, the following control protocol guarantees output synchronization ( $\mathrm{Su}$, et al., 2012; Yang, et al., 2014; Grip et al., 2012)

$u_{i}=\bar{K}_{1 i}\left(x_{i}-\Pi_{i} \zeta_{i}\right)+\Gamma_{i} \zeta_{i}$

where $\bar{K}_{1 i} \in \mathbb{R}^{m_{i} \times n_{i}}$ is the state-feedback gain which stabilizes $A_{i}+B_{i} \bar{K}_{1 i}$. Moreover, $\zeta_{i}$ is the estimate of the leader trajectory $\zeta_{0}$ for agent $i$ and is given by (Jiao el al., 2015)

$\dot{\zeta}_{i}=S \zeta_{i}+c\left[\sum_{j=1}^{N} a_{i j}\left(\zeta_{j}-\zeta_{i}\right)+g_{i}\left(\zeta_{0}-\zeta_{i}\right)\right]$

where $c$ is the coupling gain.

Remark 1: Note that the solution to the output regulator equation (4) requires the complete knowledge of the leader and the agents' dynamics. In order to obviate this requirement, a model-free distributed adaptive observer is first designed in this paper to estimate the leader's state $\zeta_{0}$. Then, a model-free off-policy reinforcement learning algorithm is developed to solve the optimal tracking problem online in real time.

\section{Distributed Adaptive Observer}

In this section, a model-free distributed adaptive observer is designed to estimate the leader's state and output for each agent.

To estimate the leader's state, the following distributed observer is used.

$\dot{\hat{\zeta}}_{i}=\hat{S}_{i} \hat{\zeta}_{i}+c\left[\sum_{j=1}^{N} a_{i j}\left(\hat{\zeta}_{j}-\hat{\zeta}_{i}\right)+g_{i}\left(\zeta_{0}-\hat{\zeta}\right)\right]$

$\hat{y}_{0 i}=\hat{R}_{i} \hat{\zeta}_{i}$ 
where $\hat{S}_{i} \in \mathbb{R}^{p \times p}$ and $\hat{R}_{i} \in \mathbb{R}^{q \times p}$ are estimations of the leader's dynamics $S$ and $R$, respectively, and $\hat{\zeta}_{i}$ and $\hat{y}_{0 i}$ are the state and output of the adaptive observer for node $i$, respectively.

The state observer estimation error is defined as

$\delta_{i}(t)=\hat{\zeta}_{i}(t)-\zeta_{0}(t)$

and the output observer estimation error is defined as

$\tilde{y}_{0 i}(t)=\hat{y}_{0 i}(t)-y_{0}(t)$

The local neighborhood observation error for node $i$ is defined as

$e_{i}=\sum_{j=1}^{N} a_{i j}\left(\hat{\zeta}_{j}-\hat{\zeta}_{i}\right)+g_{i}\left(\zeta_{0}-\hat{\zeta}_{i}\right)$

The adaptation law for $\hat{S}_{i}$ in (7) is designed as

$\dot{\hat{S}}_{\text {veci }}=\Gamma_{S i}\left(I_{p} \otimes \hat{\zeta}_{i}\right) p_{i} \sum_{j=1}^{N} a_{i j}\left(\hat{\zeta}_{j}-\hat{\zeta}_{i}\right)+g_{i}\left(\zeta_{0}-\hat{\zeta}_{i}\right)$

where $\hat{S}_{\text {vec } i}$ is a vector obtained by stacking rows of the matrix $\hat{S}_{i}^{\text {veci }}, \Gamma_{S i}>0$ and

$p_{i}=\operatorname{diag}\left(q_{i}\right)>0$

where

$q=\left[q_{1}, \ldots, q_{N}\right]^{T}=\left((L+G) \otimes I_{p}\right)^{-T} 1_{N p}$

for general directed graphs and $q \in \mathbb{R}^{N_{p}}$ is the left eigenvector of the graph Laplacian, with $1_{N p}$ vector with all entries 1 and $q_{i} \in \mathbb{R}^{p}$ for all $i \in N$. It was shown in (Zhang, Li, Qu, \& Lewis, 2015) that $p_{i}>0$.

The adaptation law for $\hat{R}_{i}$ in (8) is designed as

$\dot{\hat{R}}_{i}=\alpha\left[\sum_{j=1}^{N} a_{i j}\left(\hat{R}_{j} \hat{\zeta}_{j}-\hat{R} \hat{\zeta}_{i}\right)+g_{i}\left(y_{0}-\hat{R}_{i} \hat{\zeta}_{i}\right)\right] \hat{\zeta}_{i}^{T}$

where $\alpha>0$ is a scalar.

It is shown in the following theorem that using these update laws, the state and output observer estimation errors (9) and (10) converge if the graph has a spanning tree.

Theorem 1: Let Assumptions 1 and 2 be satisfied and the coupling gain $c$ be large enough. Then, the observer (7), (8) with the tuning laws (12), (15) ensures that

a) estimation errors (9) and (10) go to zero for detail balanced graph.

b) estimation errors (9) and (10) are uniformly ultimately bounded (UUB) for general directed graphs.

Proof. Differentiating (9), and using (1) and (7) gives the local estimation error dynamics for each observer as

$\dot{\delta}_{i}=\hat{S}_{i} \hat{\zeta}_{i}+c\left[\sum_{j=1}^{N} a_{i j}\left(\hat{\zeta}_{j}-\hat{\zeta}_{i}\right)+g_{i}\left(\zeta_{0}-\hat{\zeta}_{i}\right)\right]-S \zeta_{0}$

Adding and subtracting $S \hat{\zeta}_{i}$, the global error dynamics for all the agents in a compact form is

$\dot{\delta}=\left[I_{N} \otimes S-c(L+G) \otimes I_{p}\right] \delta+\tilde{S} \hat{\zeta}$

where $\quad \delta=\hat{\zeta}-\zeta_{0} \quad$ with $\hat{\zeta}=\left[\begin{array}{lll}\hat{\zeta}_{1}^{T} & \hat{\zeta}_{2}{ }^{T} \cdots \hat{\zeta}_{N}^{T}\end{array}\right]^{T}, \quad \zeta_{0}=$ $\left[\zeta_{0}^{T} \zeta_{0}^{T} \cdots \zeta_{0}^{T}\right]^{T}$ and $\tilde{S}=\operatorname{diag}\left(\tilde{S}_{i}\right) \quad$ with $\tilde{S}_{i}=\hat{S}_{i}-S, \forall i$ The error dynamics (17) can be rewritten as,
$\dot{\delta}=A_{S} \delta+\zeta_{M} \tilde{S}_{\text {vec }}$

where $\tilde{S}_{\text {vec }}$ is the vector representation of the matrix $\tilde{S}$, and

$A_{s}=I_{N} \otimes S-c(L+G) \otimes I_{p}$

$\zeta_{M}=\operatorname{diag}\left(I_{p} \otimes \hat{\zeta}_{1}, I_{p} \otimes \hat{\zeta}_{2}, \ldots, I_{p} \otimes \hat{\zeta}_{N}\right)^{T}$

The error dynamics matrix $A_{s}$ can be made Hurwitz for an appropriate choice of $c$, because $L+G$ is symmetric and has nonsingular and real positive eigenvalues.

Now consider the Lyapunov function

$V=\delta^{T} H_{\delta} \delta+\tilde{S}_{\text {vec }}^{T}\left(2 \Gamma_{S}^{-1}\right) \tilde{S}_{\text {vec }}$

where

$H_{\delta}=P\left((L+G) \otimes I_{p}\right)+\left((L+G) \otimes I_{p}\right)^{T} P$

with $P=\operatorname{diag}\left(p_{i}\right)$ where $p_{i}$ is defined in (13), and $\Gamma_{S}=\operatorname{diag}\left[\Gamma_{S 1} \Gamma_{S 2} \cdots \Gamma_{S N}\right]$. Note that $H_{\delta}$ is symmetric and positive definite (Zhang, Li, Qu, \& Lewis, 2015). Using (18), the derivative of (21) yields

$\dot{V}=-\delta^{T} Q \delta+\tilde{S}_{\text {vec }}^{T} \zeta_{M}^{T} H_{\delta} \delta+\delta^{T} H_{\delta} \zeta_{M} \tilde{S}_{\text {vec }}$

$+\dot{\tilde{S}}_{\text {vec }}^{T}\left(2 \Gamma_{S}^{-1}\right) \tilde{S}_{\text {vec }}+\tilde{S}_{\text {vec }}^{T}\left(2 \Gamma_{S}^{-1}\right) \dot{\tilde{S}}_{\text {vec }}$

where

$-Q=H_{\delta} A_{S}+A_{S}^{T} H_{\delta}$

The matrix $Q$ is symmetric since $H_{\delta}$ is symmetric. To show that $Q$ is positive definite, define $I_{V} \otimes S=M$ and $(L+G) \otimes I=N$, where $M$ has no eigenvalues with positive real parts, and $N$ is non-singular. Then, $Q$ becomes

$$
\begin{aligned}
& Q=-\left(P N+N^{T} P\right)(M-c N)-\left(M^{T}-c N^{T}\right)\left(P N+N^{T} P\right) \\
& =c\left(P N N+N^{T} N^{T} P\right)-\left(P N M+M^{T} N^{T} P\right) \\
& \quad+c\left(N^{T} P N+N^{T} P N\right)-\left(N^{T} P M+M^{T} P N\right)
\end{aligned}
$$

For any given Hermitian matrices $E, F$, and some constant $c$, the eigenvalue of the matrix sum is (Fulton, 2000; Strang, 2006)

$\lambda_{i+j-1}(c E-F) \leq c \lambda_{i}(E)-\lambda_{j}(F)$

for $i+j \leq N+1, i \leq N, j \leq N$. Using this inequality, one can conclude that $Q$ is positive definite if $c$ is large enough.

If the graph is detail balanced, then one has (Lewis et al., 2014)

$$
P\left((L+G) \otimes I_{p}\right)=\left((L+G) \otimes I_{p}\right)^{T} P
$$

and therefore, the global error dynamics for (12) becomes

$$
\dot{\tilde{S}}_{\text {vec }}=-\frac{1}{2} \Gamma_{S} \zeta_{M}^{T} H_{\delta} \delta=-\Gamma_{S} \zeta_{M}^{T} P\left((L+G) \otimes I_{p}\right) \delta
$$

Using (28) in (23), the Lyapunov derivative becomes

$\dot{V}=-\delta^{T} Q \delta$

Hence, the Lyapunov derivative (29) is negative semidefinite and the proof of first component of part a is completed. If the graph is not detail balanced, condition (27) may not be satisfied, by using the update law (28) the Lyapunov derivative becomes 


$$
\dot{V}=-\delta^{T} Q \delta+\tilde{S}_{\text {vec }}^{T} \zeta_{M}^{T} D \delta+\delta^{T} D \zeta_{M} \tilde{S}_{\text {vec }}
$$

where

$$
D=\left((L+G) \otimes I_{p}\right)^{T} P-P\left((L+G) \otimes I_{p}\right) \equiv O-O^{T}
$$

is skew symmetric. Using $\hat{\zeta}^{T}=\delta+\zeta_{0}$ and skew symmetry of $D$ in (30)

$$
\dot{V}=-\delta^{T} Q \delta+\underline{\zeta}_{0}^{T} \tilde{S}^{T} D \delta+\delta^{T} D \tilde{S} \underline{\zeta}_{0}
$$

which yields

$$
\dot{V} \leq-c \underline{\sigma}(Q)\|\delta\|^{2}+b_{\zeta} S_{M} \bar{\sigma}(D)\|\delta\|
$$

where $S_{M}$ is the bound on the leader dynamics estimation error, $b$ is the bound on the leader's state, $\underline{\sigma}$ and $\bar{\sigma}$ denote the minimum and maximum singular values, respectively. This completes the proof of part b. $\tilde{S}$ can be made bounded by using the projection operator in the update law (12).

To show that (10) goes to zero, note that $\delta_{i}(t) \rightarrow 0$ for detail balanced graphs, regardless of the observer output. Therefore, the convergence of $\hat{R}_{i}$ is decoupled form $\hat{S}_{i}$. When the observer state converges, using (15) one has

$$
\dot{\tilde{R}}_{i}=\dot{\hat{R}}_{i}-\dot{R}=\alpha\left[\sum_{j=1}^{N} a_{i j}\left(\hat{R}_{j}-\hat{R}_{i}\right)+g_{i}\left(R-\hat{R}_{i}\right)\right] \zeta_{0} \zeta_{0}{ }^{T}
$$

The global form of (34) yields

$$
\left.\dot{\tilde{R}}_{v e c}=-\alpha(L+G) \otimes I_{p}\right) \mathbf{z}_{0} \tilde{R}_{v e c}
$$

where $\mathbf{z}_{0}=\operatorname{diag}\left(\zeta_{0} \zeta_{0}{ }^{T}, \ldots, \zeta_{0} \zeta_{0}{ }^{T}\right)$. Based on Assumption 2, this concludes that $R_{v e c}$ and consequently $\tilde{y}_{i}$ goes to zero.

Remark 2: Note that $\underline{\sigma}(Q)$ can be made arbitrarily large by choosing a large $c$ and therefore, the observer error can be made arbitrarily small. The larger the initial error of the leader dynamics is, the larger the gain $c$ needs to be. If the graph is undirected, then $L+G$ is positive definite and thus $p_{i}$ in (12) can be identity. For other graphs, $p_{i}$ in (14) requires the knowledge of the graph. However, since $\Gamma$ in (12) is arbitrary, therefore $\Gamma_{i} p_{i}$ can also be arbitrary. Therefore, the proposed update law (12) does not need any knowledge of the graph structure.

\section{Optimal Model-free Output Regulation}

In this section, a reinforcement learning (RL) algorithm is proposed to make the agents track the leader's output. In Section 5, this RL based optimal control will be combined with the distributed adaptive synchronizer of Section 2 .

Consider the linear heterogeneous agents given by (3). The goal is to find a control policy to make the agent output $y_{i}$ in (3) follow the reference trajectory output $y_{0}$ generated by (1), (2), while minimizing a performance function. Define the discounted performance function for the system (3) as (Modares \& Lewis, 2015)

$$
V\left(x_{i}(t), u_{i}(t)\right)=\int_{t}^{\infty} e^{-\gamma_{i}^{(\tau-t)}}\left(\left(y_{i}-y_{0}\right)^{T} Q_{i}\left(y_{i}-y_{0}\right)+u_{i}^{T} W_{i} u_{i}\right) d \tau
$$

where the weight matrices $Q_{i}$ and $W_{i}$ are symmetric positive definite, and $\gamma_{i}>0$ is the discount factor.

Remark 3: The discount factor $\gamma_{i}>0$ in (36) is used to ensure that the performance function is bounded for a given control policy which assures the output regulation (Modares \& Lewis, 2015).

Consider a fixed state-feedback control policy as

$$
u_{i}=K_{1 i} x_{i}+K_{2 i} \zeta_{0}
$$

and define an augmented state as

$$
X(t)=\left\lceil x_{i}(t)^{T} \zeta_{0}^{T}\right]^{T} \in \mathbb{R}^{n_{i}+p}
$$

where $\zeta_{0}$ is given by (1). The control input (37) in terms of the augmented state (38) becomes

$u_{i}=K_{1 i} x_{i}+K_{2 i} \zeta_{0}=K_{i} X_{i}$

where $K_{i}=\left[\begin{array}{ll}K_{1 i} & K_{2 i}\end{array}\right]$. The augmented dynamics become (Modares \& Lewis, 2015)

$$
\dot{X}_{i}=T_{i} X_{i}+B_{1 i} u_{i}
$$

with

$$
T_{i}=\left[\begin{array}{cc}
A_{i} & 0 \\
0 & S
\end{array}\right], B_{1 i}=\left[\begin{array}{c}
B_{i} \\
0
\end{array}\right]
$$

Finally, the value function for a control policy in form of (39) can be written as the quadratic form (Modares \& Lewis, 2015)

$$
\begin{aligned}
V\left(X_{i}(t)\right) & =\int_{t}^{\infty} e^{-\gamma_{i}(\tau-t)} X_{i}^{T}\left(C_{1 i}{ }^{T} Q_{i} C_{1 i}+K_{i}^{T} W_{i} K_{i}\right) X_{i} d \tau \\
= & X_{i}(t)^{T} P_{i} X_{i}(t)
\end{aligned}
$$

where

$C_{1 i}=\left[\begin{array}{ll}C_{i} & -R\end{array}\right]$

with $R$ as in (2). The optimal control input is then given by $u_{i}=K_{i} X_{i}$ (Modares \& Lewis, 2015) with

$K_{i}=\left[K_{1 i}, K_{2 i}\right]=-W_{i}^{-1} B_{1 i}{ }^{T} P_{i}$

where $P_{i}$ is the solution to the discounted algebraic Riccati equation (ARE)

$T_{i}^{T} P_{i}+T_{i} P_{i}-\gamma_{i} P_{i}+C_{1 i}{ }^{T} Q_{i} C_{1 i}-P_{i} B_{1 i} W_{i}^{-1} B_{1 i}{ }^{T} P_{i}=0$

The ARE (45) is first solved for $P_{i}$, and then the optimal gain is obtained by substituting the ARE solution to (44).

Remark 4: The control gain (44) depends on the solution of the ARE (45) which requires complete knowledge of the agent's dynamics. This requirement will be obviated in Section 4.2 by designing a model-free reinforcement learning algorithm to learn online the gains (45).

\subsection{An upper bound for discount factor to assure asymptotic output regulation}

The following theorem shows that perfect output regulation is achieved if (44) is applied to the system and the discount factor is chosen small enough.

Theorem 2: Let the control input (39) with gain given by (44), (45) is applied to the system (3), then $A_{i}+B_{i} K_{1 i}$ is Hurwitz and the tracking error $e_{r i}=y_{i}-y_{0}$ goes to zero asymptotically fast if the discount factor satisfies the following condition

$\gamma_{i} \leq \gamma_{i}^{*}=2\left\|\left(B_{i} W_{i}^{-1} B_{i}^{T} Q_{i}\right)^{1 / 2}\right\|$. 
Proof. We first show that $A_{i}+B_{i} K_{1 i}$ is Hurwitz. To this end, define

$$
P_{i}=\left[\begin{array}{cc}
P_{11}^{i} & P_{12}^{i} \\
P^{i} & P_{22}^{i}
\end{array}\right]
$$

Then, using (41), for the upper left-hand side of the discounted ARE (45) one has

$$
\begin{aligned}
& A_{i}^{T} P_{11}^{i}+P_{11}^{i} A_{i}-\gamma_{i} P^{i}{ }_{11}+C_{1 i}{ }^{T} Q_{i} C_{1 i}-P_{11}^{i} B_{1 i} W_{i}^{-1} B_{1 i}{ }^{T} P^{i}{ }_{11} \\
& =0
\end{aligned}
$$

and the control gain $K_{1 i}$ becomes

$$
K_{1 i}=-W_{i}^{-1} B^{T} P_{11}^{i}
$$

It is shown in (Modares, Lewis \& Jiang, 2015) that if (47) is satisfied, then $A_{i}+B_{i} K_{1 i}$ is Hurwitz. Multiplying the left- and right- hand sides of the ARE (45) by $X_{i}^{T}$ and $X_{i}$, respectively, one has

$$
\begin{aligned}
& 2 X_{i}^{T} T_{i}^{T} P_{i} X_{i}-\gamma_{i} X_{i}^{T} P_{i} X_{i}+X_{i}^{T} C_{1 i}{ }^{T} Q_{i} C_{1 i} X_{i}- \\
& \left(P_{i} X_{i}\right)^{T} B_{1 i} W_{i}^{-1} B_{1 i}{ }^{T}\left(P_{i} X_{i}\right)=0
\end{aligned}
$$

One can see that if $P_{i} X_{i}=0$, then $X_{i}^{T} C_{1 i}{ }^{T} Q_{i} C_{1 i} X_{i}=0$. That is, the null space of $P$ is a subspace of the null space of $C_{1 i}{ }^{T} Q_{i} C_{1 i}$. This indicates that if $X_{i}^{T} P_{i} X_{i}=0$ then $X_{i}^{T} C_{1 i}^{T} Q_{i} C_{1 i} X_{i}=0$ and thus $\left(y_{i}-y_{0}\right)^{T} Q_{i}\left(y_{i}-y_{0}\right)=0$ which yields $e_{r i}=y_{i}-y_{0}=0$. Therefore, the null space of $P_{i}$ is in fact a subspace of the space in which the tracking error is zero. Now, consider the following Lyapunov function

$$
V_{i}\left(X_{i}\right)=X_{i}^{T} P_{i} X_{i} \geq 0
$$

To complete the proof, it remains to show that $\dot{V}_{i}\left(X_{i}\right)<0$ if $X_{i}^{T} P_{i} X_{i} \neq 0$ and (46) is satisfied. That is, the null space of $\stackrel{i}{P}_{i}$ is attractive. Taking the derivative of $V_{i}\left(X_{i}\right)$ gives

$$
\dot{V}_{i}\left(X_{i}\right)=X_{i}^{T}\left(P_{i} A_{c i}+A_{c i}{ }^{T} P_{i}\right) X_{i}
$$

where

$$
A_{c i}=\left[\begin{array}{cc}
A_{i}+B_{i} K_{1 i} & B_{i} K_{2 i} \\
0 & S
\end{array}\right]
$$

If condition (46) is satisfied, then $A_{i}+B_{i} K_{1 i}$ is Hurwitz. Since $S$ has all its eigenvalues on the imaginary axis, $A_{c i}$ is marginally stable and thus there exists a $Q \geq 0$ such that $\dot{V}_{i}\left(X_{i}\right)=-X_{i}^{T} Q X_{i} \leq 0$. Based on LaSalle's invariance principle, $X_{i}$ converge to the largest invariant subspace where $\dot{V}\left(X_{i}\right)=0$. Using (52), $\dot{V}\left(X_{i}\right)=0$ if $P_{i} X_{i}=0$ and this completes the proof.

\subsection{Model-free Off-policy Reinforcement Learning for Solving Optimal Output Regulation}

In order to find the optimal gain (44) without the requirement of the knowledge of the system dynamics, offpolicy IRL algorithm (Modares, Lewis \& jiang, 2015) is used in this subsection. To this end, the system dynamics (40) is first written as

$$
\dot{X}_{i}=T_{i} X_{i}+B_{1 i}\left(-K_{i}^{\kappa} X_{i}+u_{i}\right)
$$

where $T_{i}=T_{i}+B_{1 i} K_{i}^{\kappa}$.
The Bellman equation becomes (Modares \& Lewis, 2015)

$$
\begin{aligned}
& e^{-\gamma_{i} \delta t} X_{i}(t+\delta t)^{T} P_{i}^{\kappa} X_{i}(t+\delta t)-X_{i}(t)^{T} P_{i}^{\kappa} X_{i}(t)= \\
& \int_{t}^{t+\delta t} \frac{d}{d \tau}\left(e^{-\gamma_{i}(\tau-t)} X_{i}^{T} P_{i}^{\kappa} X_{i}\right) d \tau= \\
& =-\int_{t}^{t+\delta t} e^{-\gamma_{i}(\tau-t)} X_{i}^{T} Q_{i} X_{i} d \tau+ \\
& 2 \int_{t}^{t+\delta t} e^{-\gamma_{i}(\tau-t)}\left(u_{i}-K_{i}^{\kappa} X_{i}\right)^{T} W_{i} K_{i}^{\kappa+1} X_{i} d \tau \\
& \text { where } Q_{i}=C_{1 i}{ }^{T} Q_{i} C_{1 i}+\left(K_{i}^{\kappa}\right)^{T} W_{i}\left(K_{i}^{\kappa}\right) .
\end{aligned}
$$

Remark 5: The Bellman equation (55) can be solved for both the kernel matrix $P_{i}^{\kappa}$ and the improved gain $K_{i}^{\kappa+1}$, simultaneously. No knowledge of the agent and leader dynamics is required. Note that although $Q_{i}$ in the first integrand in the right-hand-side of (55) depends on the leader dynamic $R$ and the agent dynamics $C_{i}$ (see (43)), one can use only the output measurement to calculate this integrand using $X_{i}^{T} Q_{i} X_{i}=\left(y_{i}-y_{0}\right)^{T} Q_{i}\left(y_{i}-y_{0}\right)$. The leader output $y_{0}$ is not available to all agents and one can implement (55) by replacing $X_{i}^{T} Q_{i} X_{i}$ with $\left(y_{i}-\hat{y}_{0 i}\right)^{T} Q_{i}\left(y_{i}-\hat{y}_{0 i}\right)$ where $\hat{y}_{0 i}$ is the estimation of the leader output as defined in (8). It was shown in Theorem 1 that using the proposed adaptation law assures $\hat{y}_{0 i} \rightarrow y_{0}$. The additional details for implementation of Algorithm 1 are given in Section 5 .

The following model-free Algorithm 1 uses the above Bellman equation to simultaneously solve the ARE equation (45) and find the gain (44).

\section{Algorithm 1. Online Off-policy IRL State-feedback algorithm}

1. Initialization: Start with a control policy $u_{i}^{\kappa}=K_{i}^{0} X_{i}+e$, where $K_{i}{ }^{\kappa}$ is stabilizing, $e$ is the probing noise and $\kappa=0$

2. Solve the following Bellman equation for $P_{i}^{\kappa}$ and $K_{i}^{\kappa+1}$ simultaneously.

$$
\begin{aligned}
& e^{-\gamma_{i} \delta t} X_{i}(t+\delta t)^{T} P_{i}^{\kappa} X_{i}(t+\delta t)-X_{i}(t)^{T} P_{i}^{\kappa} X_{i}(t)= \\
& -\int_{t}^{t+\delta t} e^{-\gamma_{i}(\tau-t)}\left(y_{i}-y_{0}\right)^{T} Q_{i}\left(y_{i}-y_{0}\right) d \tau+ \\
& 2 \int_{t}^{t+\delta t} e^{-\gamma_{i}(\tau-t)}\left(u_{i}-K_{i}^{\kappa} X_{i}\right)^{T} W_{i} K_{i}^{\kappa+1} X_{i} d \tau
\end{aligned}
$$

3. Stop if convergence is achieved, otherwise set $\kappa=\kappa+1$ and go to 2 .

\section{On convergence set $K_{i}=K_{i}^{\kappa}$}

In Algorithm 1, the control policy which is applied to the systems, i.e. $u_{i}$, can be a fixed stabilizing policy. The data which is gathered by applying this fixed policy to the system is then used in (56) to find the matrix $P_{i}^{\kappa}$ and the improved policy $u_{i}^{\kappa+1}=K_{i}^{\kappa+1} X_{i}$. This corresponds to an updated new policy $u_{i}=K_{i}^{i} X_{i}$.

Remark 6: This unique solution for $P_{i}^{\kappa}, K_{i}^{\kappa+1}$ in (56) can be uniquely determined using the least squares (LS) technique under some persistence of excitation (PE) 
condition (Jiang \& Jaing, 2012). Enough sample are collected by applying a fixed control input to each agent before (56) can be solved for a unique solution using LS under some persistence of excitation condition.

\section{Optimal Model-free Output Regulation for a Multi- agent Heterogeneous System}

In this section the distributed observer and the optimal tracking control from Sections 4 and 5 are combined to solve Problem 1 without requiring any knowledge of the system dynamics. The optimal control law (39) in Section 4 depends on the leader's state $\zeta_{0}$. However, the adaptive observer (7) and the corresponding update law (12) provides the leader estimation for each agent. Therefore, by replacing the leader's state $\zeta_{0}$ in (39) with the observer's state $\hat{\zeta}_{i}$, the modified distributed optimal controller for each agent yields

$$
u_{i}=K_{1 i} x_{i}+K_{2 i} \hat{\zeta}_{i} \equiv K_{i} \hat{X}_{i}
$$

where $\hat{X}(t)=\left[\begin{array}{ll}x_{i}^{T} & \hat{\zeta}_{i}^{T}\end{array}\right]^{T}$, with $x_{i}$ the agent's local state and $\hat{\zeta}_{i}$ the observer's state given by (7). Moreover, the control gain $K_{i}$ is given by (44), (45). Algorithm 1 with $\hat{X}(t)=\left[\begin{array}{ll}x_{i}^{T} & \hat{\zeta}_{i}^{T}\end{array}\right]^{T}$ in place of $X(t)$ and replacing $X_{i}^{T} Q_{i} X_{i}$ by $\left(y_{i}-\hat{y}_{0 i}\right)^{T} Q_{i}\left(y_{i}-\hat{y}_{0 i}\right)$ can be used to find $K_{i}$ without requiring any knowledge of the agent's dynamics $\left(A_{i}, B_{i}, C_{i}\right)$ or the leader's dynamic matrix $S$.

The following theorem shows that the control protocol (57), guarantees output synchronization of multi-agent heterogeneous systems.

Theorem 3: Consider the distributed adaptive synchronizer (7) and (8) and the optimal tracking controller (57) with $K_{i}$ given by (44), (45). Then the output synchronization problem is solved, provided that $c$ in (7) is sufficiently large and the discount factor $\gamma_{i}$ is less than the bound (46).

Proof: Using the control law (57), consider the augmented dynamics for a single agent

$$
\left[\begin{array}{c}
\dot{x}_{i} \\
\dot{\hat{\zeta}} \\
\zeta_{i}
\end{array}\right]=\left[\begin{array}{cc}
A_{i}+B_{i} K_{1 i} & B_{i} K_{2 i} \\
0 & \hat{S}_{i}
\end{array}\right]\left[\begin{array}{c}
x_{i} \\
\hat{\zeta}_{i}
\end{array}\right]+\left[\begin{array}{c}
0 \\
e_{i}
\end{array}\right]
$$

along with the adaptive law given by (12) as

$$
\dot{\hat{S}}_{\text {veci }}=-\Gamma_{S i} p_{i}\left(I_{q} \otimes \hat{\zeta}_{i}\right) e_{i}
$$

where $e_{i}$ is defined in (11). Due to the block-triangular structure, the observer dynamics is independent of the agent state $x_{i}$, and thus based on the separation principle the observer and the tracking control can be designed independent of each other. It is shown in Theorem 1 that for detail balanced graphs, if the coupling gain is large enough, then $\hat{\zeta}_{i}(t)-\zeta_{a}(t) \rightarrow 0, \forall i=1, \cdots, N$. For any full rank matrix $R, R \zeta_{i}(t)-R \zeta_{0}(t) \rightarrow 0$, i.e., $R \hat{\zeta}(t)-y_{0}(t) \rightarrow 0$. Based on Theorem 2, if the discount factor is small enough, then $\quad y_{i}(t)-R \hat{\zeta}(t) \rightarrow 0, \quad$ and therefore $y_{i}(t)-y_{0}(t) \rightarrow 0, \forall i$. For a generic digraph this error can be made arbitrarily small.

It is well known in the literature that solving the output synchronization problem requires the solution to the output regulation equations (4) (Huang, 20004). In the previous theorem, it was shown that the proposed approach does not require the explicit solution of the output regulator equations (4). However, the following lemma demonstrates that our proposed approach implicitly solves this equation without actually doing so.

Lemma 1: Consider the network of heterogeneous multiagent systems (3), and the leader (1). Then, the control law (57) implicitly solves the output regulation equations (4). Moreover, if the gain $K_{1 i}$ in (57) is the same as $\bar{K}_{1 i}$ in (5), then $K_{2 i}=\Gamma_{i}-K_{1 i} \Pi_{i}$. Finally, the relationship between the solution to output regulator equations (4) and the ARE (45) is given by

$\Pi_{i}=-\left(P_{11}^{i}\right)^{-1} P_{12}^{i}$

where $P_{11}^{i}$ and $P_{12}^{i}$ are defined as (47).

Proof: From Theorem 2, the control law (57) obtained using Algorithm 1, stabilizes the system (3), i.e., $\left(A_{i}+B_{i} K_{1 i}\right)$ is Hurwitz. Therefore, based on Assumption 2, there exists a unique matrix $\Pi_{i} \in \mathbb{R}^{n_{i} \times p}$ that satisfies

$$
\left(A_{i}+B_{i} K_{1 i}\right) \Pi_{i}+B_{i} K_{2 i}=\Pi_{i} S
$$

This is a Sylvester equation and the existence of the solution $\Pi_{i}$ is guaranteed since $\sigma(S) \cap \sigma\left(A_{i}+B_{i} K_{1 i}\right) \in \varnothing($ Knobloch, Isidori \& Flockerzi, 1993). Also, based on Theorems 1,2 and 3, an appropriate value of $\gamma$ achieves the output regulation for control law (57), i.e.,

$\lim _{t \rightarrow \infty} y_{i}(t)-y_{0}(t)=\lim _{t \rightarrow \infty} C_{i} x_{i}(t)-R \zeta_{0}(t)=0$

Therefore, there exists an invariant zero-error subspace $\vartheta_{i}=\left\{X_{i} \mid x_{i}=\Pi_{i} \zeta_{0}\right\}$. Define the state transformation $\bar{x}_{i}=x_{i}-\Pi_{i}^{i} \zeta_{0}$. Then, the dynamics of the new state $\bar{x}_{i}$ is

$\dot{\bar{x}}_{i}=\dot{x}_{i}-\Pi_{i} \dot{\zeta}_{0}=A_{i} x_{i}+B_{i} K_{1 i} x_{i}+B_{i} K_{2 i} \zeta_{0}-\Pi_{i} S \zeta_{0}$ $=\left(A_{i}+B_{i} K_{1 i}\right) \bar{x}_{i}$

Thus, $\lim \bar{x}_{i}=0$, since $\left(A_{i}+B_{i} K_{1 i}\right)$ is Hurwitz. On the other hand, from Theorem 2, one has

$\lim _{t \rightarrow \infty} C_{i} x_{i}(t)-R \zeta_{0}(t)=\lim _{t \rightarrow \infty} C_{i} \bar{x}_{i}(t)+\lim _{t \rightarrow \infty}\left(C_{i} \Pi_{i}-R\right) \zeta_{0}(t)=0$

Since $\zeta_{0}(t)$ is obtained from a marginally stable system (Assumption 2), this implies $C_{i} \Pi_{i}-R=0$. Using the transformation $\Gamma_{i}=K_{2 i}+K_{1 i} \Pi_{i}$ in (61) along with (64) gives (4).

To show that (60) is satisfied, based on Theorem 2, the tracking error is zero if the augmented state trajectory of the null space of the ARE solution. That is, the synchronization error is zero if

$P_{i} X_{i}=\left[\begin{array}{cc}P_{11}^{i} & P_{12}^{i} \\ P^{i} & P^{i}\end{array}\right]\left[\begin{array}{l}x_{i} \\ \zeta_{0}\end{array}\right]=0$

which gives

$x_{i}=-\left(P^{i}{ }_{11}\right)^{-1} P^{i}{ }_{12} \zeta_{0}$

Since the zero-error subspace is invariant, one has $\dot{x}_{i}=-\left(P^{i}{ }_{11}\right)^{-1} P^{i}{ }_{12} \dot{\zeta}_{0}$. Using the control protocol (37) and the dynamics (3) and (1) this gives

$\left(A_{i}+B_{i} K_{1 i}\right) x_{i}+B_{i} K_{2 i} \zeta_{0}=-\left(P_{11}^{i}\right)^{-1} P_{12}^{i} S \zeta_{0}$ 
Using (60), this gives equation (61) with $\Pi_{i}=-\left(P_{11}^{i}\right)^{-1} P_{12}^{i}$ and thus the proof is completed.

\section{Simulation Results}

In this section, we provide a detailed simulation analysis of the proposed adaptive optimal output synchronization approach. We choose the leader to be sinusoidal trajectory generator and its dynamics is given by

$\dot{\zeta}_{0}=\left(\begin{array}{cc}0 & 2 \\ -2 & 0\end{array}\right) \zeta_{0}, y_{0}=\left(\begin{array}{ll}1 & 0\end{array}\right) \zeta_{0}$

The heterogeneous followers are given by (3) for $i=1 \cdots 4$ and their dynamics is

$A_{1}=0, B_{1}=10, C_{1}=1$

$A_{2}=\left(\begin{array}{ll}0 & 1 \\ 0 & 0\end{array}\right), B_{2}=\left(\begin{array}{l}0 \\ 5\end{array}\right), C_{2}=(1$

$A_{3}=\left(\begin{array}{cc}0 & 1 \\ -1 & 0\end{array}\right), B_{3}=\left(\begin{array}{l}0 \\ 2\end{array}\right), C_{3}=\left(\begin{array}{ll}1 & 0\end{array}\right)$

$A_{4}=\left(\begin{array}{ccc}0 & 0 & 0 \\ 0 & 0 & 1 \\ 0 & -1 & 0\end{array}\right), B_{4}=\left(\begin{array}{l}5 \\ 0 \\ 10\end{array}\right), C_{4}=\left(\begin{array}{lll}1 & 1 & 0\end{array}\right)$

The underlying communication network of heterogeneous systems is given in Fig. 1 .

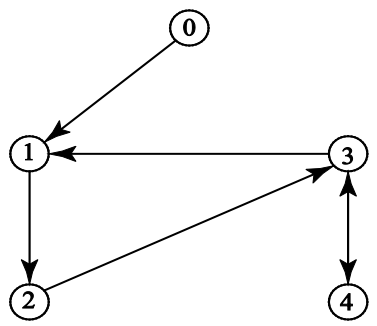

Fig.1: Communication graph for the heterogeneous systems.

The distributed observer (7), (12) is implemented for $i=1 \cdots 4$. The observer and adaptive gains are chosen as $c=25, \Gamma_{s i}=15$. The initial leader's state is chosen as $\zeta_{0}(0)=\left(\begin{array}{ll}1 & 1\end{array}\right)^{T}$. The error between observer and leader's state is given in Fig.2 and 3. It can be seen from these figures that the introduced distributed observer converges to the leader's state.

The solution of the output regulator equation (4) for the given heterogeneous systems (69) is

$$
\begin{aligned}
& \Pi_{1}=\left(\begin{array}{ll}
0 & 1
\end{array}\right), \Gamma_{1}=\left(\begin{array}{ll}
0 & 0.2
\end{array}\right) \\
& \Pi_{2}=\left(\begin{array}{ll}
1 & 0 \\
0 & 2
\end{array}\right), \Gamma_{2}=\left(\begin{array}{ll}
-0.8 & 0
\end{array}\right) \\
& \Pi_{3}=\left(\begin{array}{ll}
1 & 0 \\
0 & 2
\end{array}\right), \Gamma_{3}=\left(\begin{array}{ll}
-1.5 & 0
\end{array}\right) \\
& \Pi_{4}=\left(\begin{array}{cc}
0.36 & 0.48 \\
0.64 & -0.48 \\
0.96 & 1.28
\end{array}\right), \Gamma_{4}=\left(\begin{array}{ll}
-0.192 & 0.144
\end{array}\right)
\end{aligned}
$$

For the following choice of the weight matrices $\mathrm{Q}$ and $\mathrm{R}$, the resulting optimal state feedback gain using LQR method for (69) is
$Q_{1}=100, R_{1}=1, K_{11}=-10$

$Q_{2}=100 I, R_{2}=1, K_{12}=\left(\begin{array}{ll}-10 & -10.19\end{array}\right)$

$Q_{3}=100 I, R_{3}=1, K_{13}=\left(\begin{array}{ll}-9.51 & -10.46\end{array}\right)$

$Q_{4}=100 I, R_{4}=1, K_{14}=\left(\begin{array}{lll}-10 & -12.66 & -6.29\end{array}\right)$

Using (70) and (71), the local optimal output regulator control (5) can be solved. Instead, the tracking control is obtained online by using the Algorithm 2. The evaluation of learned optimal tracking control along with the adaptive observer for the given multi-agent heterogeneous network is in Fig.4.

It can be seen from these results that the introduced approach implicitly solves the output regulator equations (4) and solves problem 1 without requiring any knowledge of either the agent's system matrices $\left(A_{i}, B_{i}, C_{i}\right)$ or the leader's dynamic matrix $S$.

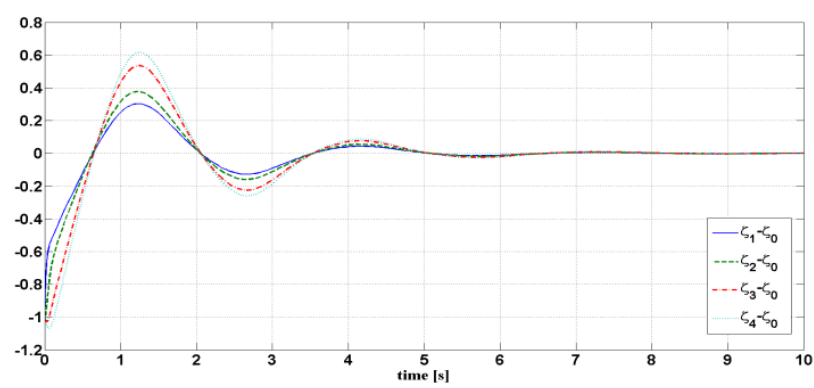

Fig.2: Error between adaptive observer and leader's trajectory for state 1 .

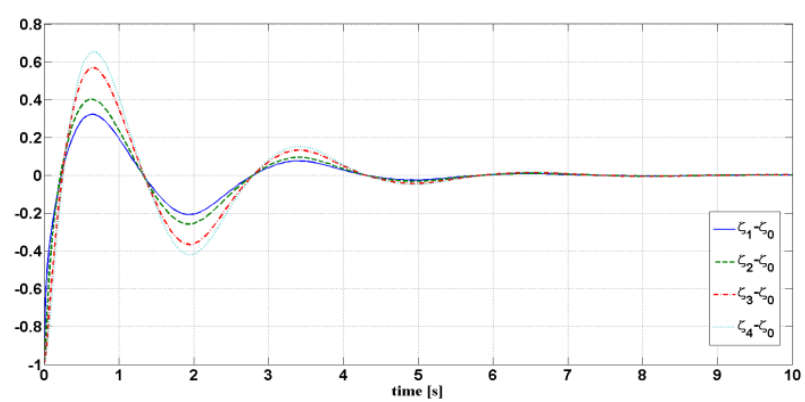

Fig.3: Error between adaptive observer and leader's trajectory for state 2 .

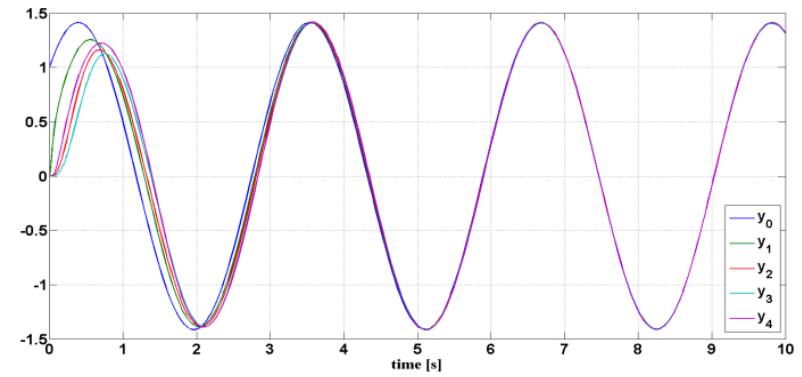

Fig.4: Evaluation of the learned controller along with adaptive observer for all 4 heterogeneous agents.

\section{Conclusion}

A novel approach is provided to design a model-free output synchronizer for heterogeneous multi-agent systems. A local discounted performance function is defined for each agent that penalizes its own control effort and its tracking error. It is shown that minimizing these performance functions leads to solving AREs. It is also shown that the solutions obtained by solving AREs guarantees 
synchronization, provided that the discount factor is small enough. An adaptive distributed observer is designed to estimate the leader's state and reinforcement learning is used to solve the AREs without requiring any knowledge of the dynamics of the agents. A simulation example is provided to show that the proposed approach in fact implicitly solves the output regulator equation for each agent.

\section{Acknowledgement}

This work is supported by NSF grant ECCS-1405173, ONR grant N00014-13-1-0562, ONR grant N000141410718, ARO grant W911NF-11-D-0001, China NNSF grant 61120106011, and China Education Ministry Project 111 (No.B08015).

\section{REFERENCES}

Jadbabaie, A., Lin, J., \& Morse, A. S. (2003). Coordination of groups of mobile autonomous agents using nearest neighbor rules. IEEE Transaction on Automatic Control, 48(6), 9881001.

Lewis, F. L., Zhang, H., Hengster-Movric,K., \& Das, A. (2014). Cooperative control of multi-agent systems: optimal and adaptive design approaches. Springer-Verlag.

Olfati-Saber, R., \& Murray, R. M. (2004). Consensus problems in networks of agents with switching topology and time-delays. IEEE Transaction on Automatic Control, 49(9), 1520-1533.

Ren, W., Beard, R. W., \& Atkins, E. M. (2007). Information consensus in multivehicle cooperative control. IEEE Control Systems Magazine, 27, 71-82.

Ren, W., \& Beard, R. W. (2008). Distributed consensus in multivehicle cooperative control. Springer-Verlag, London.

Lunze, J. (2012). Synchronization of heterogeneous agents. IEEE Transaction on Automatic Control, 57(11), 2885-2890.

Wieland, P., Sepulchre, R., \& Allgöwer, F. (2011). An internal model principle is necessary and sufficient for linear output synchronization. Automatica, 47, 1068-1074.

Huang, J., \& Chen, Z. (2004). A General framework for tackling the output regulation problem. IEEE Transaction on Automatic Control, 56(8), 1948-1952.

De Persis, C. and Jayawardhana, B. (2014). On the internal model principle in the coordination of nonlinear systems. IEEE Transactions on Control of Network Systems, 1(3), 272-282.

Yang, T., Saberi, A., Stoorvogel, A. A., \& Grip, H. F. (2014). Output synchronization for heterogeneous networks of introspective right invertible agents. International journal of robust and nonlinear control, 24(13), 1821-1844.

Peymani, E., Grip, H. F., Saberi, A., Wang, X., \& Fosson, T. I. (2014). H-infinity almost output synchronization of heterogeneous networks of introspective agents under external disturbances. Automatica, 50(4), 1026-1036

Huang, C., and Ye, X., Hong, Y., Wang, X. and Jiang, Z.P. (2013). Distributed output regulation of leader-follower multi-agent systems. International Journal of Robust and Nonlinear Control, 23(1), 48-66.

Xiang, J., Wei, W., \& Li, Y. (2009). Synchronized output regulation of linear networked systems. IEEE Transaction on Automatic Control, 54(6), 1336-1341.

Su, Y., Hong, Y., \& Huang, J. (2013). A general result on the cooperative robust output regulation for linear uncertain multiagent systems. IEEE Transaction on Automatic Control, 58(5), 1275-1279.

Das, A., \& Lewis, F. L. (2010). Distributed adaptive control for synchronization of unknown nonlinear networked systems. Automatica, 46(12), 2014-2021.

Ding, Z. (2015). Adaptive consensus output regulation of a class of nonlinear systems with unknown high-frequency gain. Automatica, 51, 348-355.

Zhang, H., Lewis, F. L., \& Das, A. (2011). Optimal design for synchronization of cooperative systems: state feedback, observer and output feedback. IEEE Transaction on Automatic Control, 56(8), 1948-1952.

Vamvoudakis, K. G., Lewis, F.L., \& Hudas, G.R. (2012). Multiagent differential graphical games: Online adaptive learning solution for synchronization with optimality. Automatica, 48(8), 1581-1661.

Cao, Y., \& Ren, W. (2010). Optimal linear-consensus algorithms: An LQR perspective. IEEE Transactions on Systems, Man, and Cybernetics, Part. B, Cybernetics, 40(3), 819-830.

Zhang, H., Feng, T., Liang, H., \& Luo, Y. (2015). LQR-based optimal distributed cooperative design for linear discrete-time multiagen systems. IEEE Transaction on Neural Networks and Learning Systems, in press.

Lakshmanan, H., \& Farias, D.P. (2006). Decentralized Approximate Dynamic Programming for Dynamic Networks of Agents. In Proc. Amer. control conf. pp. 1648-1653.

Chang, H.S. (2009). Decentralized Learning in Finite Markov Chains: Revisited. IEEE Transactions on Automatic Control, 54(7), 1648-1653.

Busoniu, L. Babuska, R. \& De Schutter, B. (2008). A Comprehensive Survey of Multiagent Reinforcement Learning. IEEE Transactions on Systems, Man, and Cybernetics. Part C: Applications and Reviews, 38(2), 156172.

Hong, Y. Chen, G., \& Bushnell, L. (2008). Distributed observers design for leader-following control of multi-agent networks. Automatica, 44, 846-850.

Hong, Y. Hu, J., \& Gao, L. (2008). Tracking control for multiagent consensus with an active leader and variable topology," Automatica, 42, 1177-1182.

Li, Z., Duan, Z., Chen, G., \& Huang, L. (2008). Consensus of multiagent systems and synchronization of complex networks: a unified viewpoint. IEEE Transactions on Circuits and Systems 1, 57, 213-224.

Grip, H.F., Yang, T., Saberi, A., \&. Stoorvogel, A.A. (2012). Output synchronization for heterogeneous networks of nonintrospective agents. Automatica, 48, 2444-2453.

Jiao, Q., Liu, S., Lewis, F.L., Xu, S., \& Xie, L. (2015). A Riccati equation approach to cooperative output regulation of heterogeneous multi-agent systems. Chinese Control Conference (CCC), pp. 7130-7135.

Zhang, H.W., Li, Z.K., Qu, Z.H., \& Lewis, F.L. (2015). On constructing lyapunov functions for multi-agent systems. Automatica, 58, 39-42.

Fulton, W. (2000). Eigenvalues, invariant factors, highest weights, and Schubert calculus. Bulletin of the American Mathematical Society, 37(3), 209-249.

Strang, G. (2006). Linear algebra and its applications. Thomson, Brooks/Cole Belmont, CA 2006.

Modares, H. \&. Lewis, F.L. (2014). Linear quadratic tracking control of partially unknown continuous time systems using reinforcement learning, IEEE Transactions on Automatic Control, 59(11), 3051-3056.

Modares, H., Lewis, F.L., \& Jiang, Z.P. (2015). H-inf Tracking Control of Completely-unknown Continuous-time Systems. IEEE Transactions on Neural Networks and Learning Systems, in press.

Jiang, Y., \& Jiang, Z.P. (2012). Computational adaptive optimal control for continuous-time linear systems with completely unknown dynamics", Automatica, 48(10), 2699 -2704.

Huang, J. (2004). Nonlinear output regulation. SIAM Press, Vol 1, Philadelphia 2004.

Knobloch, H.W., Isidori, A., \& Flockerzi, D. (1993). Topics in control theory. Springer, 1993.

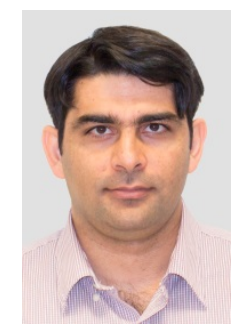

Hamidreza Modares received the B.S. degree from the University of Tehran, Tehran, Iran, in 2004, the M.S. degree from the Shahrood University of Technology, Shahrood, Iran, in 2006, and 
the Ph.D. degree from The University of Texas at Arlington (UTA), Arlington, TX, USA, in 2015. He was a Senior Lecturer with the Shahrood University of Technology, from 2006 to 2009. He is currently a Faculty Research Associate with the UTA. His current research interests include cyberphysical systems, reinforcement learning, distributed control, robotics, and pattern recognition. He has received best paper award from 2015 IEEE International Symposium on Resilient Control Systems, Stelmakh outstanding student research award from department of electrical engineering, UTA, 2015, and Summer Dissertation Fellowship, UTA, 2015

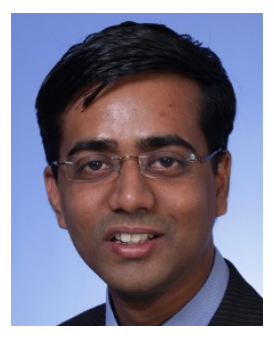

Subramanya P. Nageshrao received the B.E. degree in electronics and communication from Visvesvaraya Technological University, Belgaum, India, in 2005, M.S. degree in mechatronics from Technische Universität Hamburg-Harburg, Hamburg, Germany, in 2011, and the Ph.D. degree from Delft University of Technology, Delft, The Netherlands in 2016.

From 2005 to 2009, he was a Software Engineer with Bosch Ltd., Bangalore, India. His current research interests include nonlinear control, distributed control, machine learning particularly reinforcement learning and its applications for mechatronic and robotic systems.

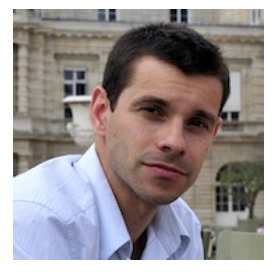

Gabriel A. D. Lopes received the M.Sc. and Ph.D. degrees in electric engineering and computer science from the University of Michigan, Ann Arbor, in 2003 and 2007, respectively. From 2005 to 2008 , he was with the GRASP laboratory, University of Pennsylvania, Philadelphia. Currently, he is an assistant professor at the Delft Center for Systems and Control, Delft University of Technology, The Netherlands. His current research interests include non-linear control, machine learning, discrete-event systems, and robotics.

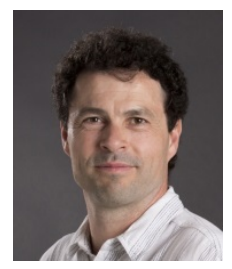

R. Babuška received the M.Sc. (Hons.) degree in control engineering from the Czech Technical University in Prague, in 1990, and the Ph.D. (cum laude) degree from the Delft University of Technology (TU Delft), the Netherlands, in 1997. He has had faculty appointments with the Czech Technical University in Prague and with the Electrical Engineering Faculty, TU Delft.

Currently, he is a Professor of Intelligent Control and Robotics at the Delft Center for Systems and Control. He is also the director of the TU Delft Robotics Institute. His current research interests include reinforcement learning, neural and fuzzy systems, nonlinear identification, stateestimation, model-based and adaptive control and dynamic multi-agent systems. He has been involved in the applications of these techniques in the fields of robotics, mechatronics, and aerospace.

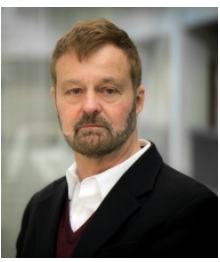

F.L. Lewis, Member, National Academy of Inventors. Fellow IEEE, Fellow IFAC, Fellow U.K. Institute of Measurement \& Control, PE Texas, U.K. Chartered Engineer. UTA Distinguished Scholar Professor, UTA Distinguished Teaching Professor, and MoncriefO'Donnell Chair at the University of Texas at Arlington Research Institute. Qian Ren Thousand Talents Consulting Professor, Northeastern University, Shenyang, China. He obtained the Bachelor's Degree in Physics/EE and the MSEE at Rice University, the MS in Aeronautical Engineering from Univ. W. Florida, and the Ph.D. at Ga. Tech. He works in feedback control, intelligent systems, cooperative control systems, and nonlinear systems. $\mathrm{He}$ is author of 7 U.S. patents, numerous journal special issues, journal papers, and 20 books, including Optimal Control, Aircraft Control, Optimal Estimation, and Robot Manipulator Control which are used as university textbooks worldwide. He received the Fulbright Research Award, NSF Research Initiation Grant, ASEE Terman Award, Int. Neural Network Soc. Gabor Award, U.K. Inst Measurement \& Control Honeywell Field Engineering Medal, IEEE Computational Intelligence Society Neural Networks Pioneer Award, AIAA Intelligent Systems Award. Received Outstanding Service Award from Dallas IEEE Section, selected as Engineer of the year by Ft. Worth IEEE Section. Was listed in Ft. Worth Business Press Top 200 Leaders in Manufacturing. Texas Regents Outstanding Teaching Award 2013. He is Distinguished Visiting Professor at Nanjing University of Science \& Technology and Project 111 Professor at Northeastern University in Shenyang, China. Founding Member of the Board of Governors of the Mediterranean Control Association. 